\title{
SCALED ENFLO TYPE IS EQUIVALENT TO RADEMACHER TYPE
}

\author{
MANOR MENDEL AND ASSAF NAOR
}

\begin{abstract}
We introduce the notion of the scaled Enflo type of a metric space, and show that for Banach spaces, scaled Enflo type $p$ is equivalent to Rademacher type $p$.
\end{abstract}

\section{Introduction}

A Banach space $X$ is said to have Rademacher type $p>0$ (see [7]) if there exists a constant $T<\infty$ such that for every $n \in \mathbb{N}$ and $x_{1}, \ldots, x_{n} \in X$,

$$
\mathbb{E}_{\varepsilon}\left\|\sum_{j=1}^{n} \varepsilon_{j} x_{j}\right\|_{X}^{p} \leqslant T^{p} \sum_{j=1}^{n}\left\|x_{j}\right\|_{X}^{p},
$$

where here, and in what follows, $\mathbb{E}_{\varepsilon}$ denotes the expectation with respect to uniformly chosen $\varepsilon=\left(\varepsilon_{1}, \ldots, \varepsilon_{n}\right) \in\{-1,1\}^{n}$. The infimum over all constants $T$ for which (1.1) holds is denoted by $T_{p}(X)$.

An important theorem of Ribe [12] states that Banach spaces which are uniformly homeomorphic must have the same isomorphic local properties. In other words, any property which remains valid under linear isomorphisms, and whose definition involves statements about finitely many vectors, is preserved under bijections which are uniformly continuous in both directions. This fact suggests that local properties of Banach spaces have a purely metric reformulation. In the past three decades this point of view fuelled research on the metric structure of Banach spaces, and led to the development of analogs of the local theory of Banach spaces in the context of general metric spaces. We refer to the discussion in $[\mathbf{2}, \mathbf{8}, \mathbf{9}]$ and the references therein for more information on this topic. In particular, as explained in the above references, motivated by the search for concrete versions of Ribe's theorem for various fundamental local properties of Banach spaces, several researchers proposed non-linear notions of type, which make sense in the setting of arbitrary metric spaces (see $[\mathbf{5}, \mathbf{3}, \mathbf{1}]$ ). Specifically, following Enflo [5], we say that a metric space $\left(\mathcal{M}, d_{\mathcal{M}}\right)$ has Enflo type $p$ if there exists a constant $K$ such that for every $n \in \mathbb{N}$ and every mapping $f:\{-1,1\}^{n} \rightarrow \mathcal{M}$,

$$
\begin{aligned}
& \mathbb{E}_{\varepsilon} d_{\mathcal{M}}(f(\varepsilon), f(-\varepsilon))^{p} \\
& \quad \leqslant K^{p} \sum_{j=1}^{n} \mathbb{E}_{\varepsilon} d_{\mathcal{M}}\left(f\left(\varepsilon_{1}, \ldots, \varepsilon_{j-1}, \varepsilon_{j}, \varepsilon_{j+1}, \ldots, \varepsilon_{n}\right), f\left(\varepsilon_{1}, \ldots, \varepsilon_{j-1},-\varepsilon_{j}, \varepsilon_{j+1}, \ldots, \varepsilon_{n}\right)\right)^{p} .
\end{aligned}
$$

For Banach spaces, (1.1) follows from (1.2) if we consider the function $\varepsilon \mapsto \sum_{j=1}^{n} \varepsilon_{j} x_{j}$. The question of whether, in the category of Banach spaces, Rademacher type $p$ implies Enflo type $p$ was posed by Enflo in [5], and in full generality remains open. In [11], Pisier showed that if a Banach space has Rademacher type $p$, then it has Enflo type $p^{\prime}$ for every $p^{\prime}<p$ (see also the work of Bourgain, Milman and Wolfson [3] for a similar result, which holds for another notion of non-linear type). In [10] it is shown that for UMD Banach spaces (see [4] for a discussion of this notion) Rademacher type $p$ is equivalent to Enflo type $p$.

Received 10 June 2005; revised 10 May 2006; published online 4 May 2007.

2000 Mathematics Subject Classification 46B20, 51F99. 
Motivated by our recent work on metric cotype [8], we introduce below the notion of the scaled Enflo type of a metric space (which is, in a sense, 'opposite' to the notion of metric cotype defined in [8]), and we show that for Banach spaces, scaled Enflo type $p$ is equivalent to Rademacher type $p$. This settles the long-standing problem of finding a purely metric formulation of the notion of type (though Enflo's problem described above remains open). Modulo some of the results of [8], the proof of our main theorem is very simple.

Definition 1. Let $\left(\mathcal{M}, d_{\mathcal{M}}\right)$ be a metric space, and let $p>0$. We say that $\mathcal{M}$ has scaled Enflo type $p$ with constant $\tau$ if for every integer $n$ there exists an even integer $m$ such that for every $f: \mathbb{Z}_{m}^{n} \rightarrow \mathcal{M}$

$$
\mathbb{E}_{\varepsilon} \int_{\mathbb{Z}_{m}^{n}} d_{\mathcal{M}}\left(f\left(x+\frac{m}{2} \varepsilon\right), f(x)\right)^{p} d \mu(x) \leqslant \tau^{p} m^{p} \sum_{j=1}^{n} \int_{\mathbb{Z}_{m}^{n}} d_{\mathcal{M}}\left(f\left(x+e_{j}\right), f(x)\right)^{p} d \mu(x),
$$

where $\mu$ is the uniform probability measure on $\mathbb{Z}_{m}^{n}$, and $\left\{e_{j}\right\}_{j=1}^{n}$ is the standard basis of $\mathbb{R}^{n}$. The infimum over all constants $\tau$ for which (1.3) holds is denoted by $\tau_{p}(\mathcal{M})$.

Theorem 1.1. Let $X$ be a Banach space and let $p \in[1,2]$. Then $X$ has Rademacher type $p$ if and only if $X$ has scaled Enflo type $p$. More precisely,

$$
\frac{1}{2 \pi} T_{p}(X) \leqslant \tau_{p}(X) \leqslant 5 T_{p}(X)
$$

The heart of Theorem 1.1 is the upper bound on $\tau_{p}(X)$ in terms of the Rademacher type $p$ constant $T_{p}(X)$ (the lower bound in Theorem 1.1 is easy to prove - see Lemma 2.1 below). If $f$ in (1.3) were a 'linear mapping', then (1.3) would trivially imply (1.1). Therefore in the proof of Theorem 1.1 we apply (1.1) to a 'smoothed' version of $f$ obtained by convolving it with the normalized indicator of a large ball in $\mathbb{Z}_{m}^{n}$. However, such an averaging necessarily results in a 'loss of information', which makes it difficult to relate the bound on the averaged $f$ obtained from (1.1) to the original function $f$. This problem is overcome by increasing the value of $m$, and is precisely why we need to scale by a factor of $m / 2$ in (1.3). The details of this argument, which relies in part on some technical estimates from [8], are presented in Section 2.

REMARK 1. If a metric space has Enflo type $p$, then it also has scaled Enflo type $p$. This follows from a straightforward modification of [8, Lemma 2.4]. We do not know whether scaled Enflo type $p$ implies Enflo type $p$. In the category of Banach spaces, a positive answer to this question would show that Enflo type $p$ is equivalent to Rademacher type $p$, positively resolving Enflo's problem [5]. We do know that for Banach spaces, scaled Enflo type $p$ implies Enflo type $p^{\prime}$ for all $p^{\prime}<p$, and that scaled Enflo type $p$ and Enflo type $p$ coincide for UMD Banach spaces.

REMARK 2. The idea of scaling by $m / 2$ in the definition of scaled Enflo type originates from the definition of metric cotype introduced in [8], which involves a similar scaling procedure. In the case of non-linear type it is possible that this scaling is not necessary (that is, that Enflo type is equivalent to Rademacher type). However, as shown in [8], in the context of metric cotype the scaling is necessary; we refer to [8] for more details.

\section{Proof of Theorem 1.1}

We start by showing that scaled Enflo type $p$ implies Rademacher type $p$.

Lemma 2.1. Let $X$ be a Banach space, and let $p \in[1,2]$. Then $T_{p}(X) \leqslant 2 \pi \cdot \tau_{p}(X)$. 
Proof. Let $X$ be a Banach space, and assume that $\tau_{p}(X)<\infty$ for some $p \in[1,2]$. Fix $\tau>\tau_{p}(X), v_{1}, \ldots, v_{n} \in X$, and let $m$ be an even integer. Define $f: \mathbb{Z}_{m}^{n} \rightarrow X$ by

$$
f\left(x_{1}, \ldots, x_{n}\right)=\sum_{j=1}^{n} e^{2 \pi i x_{j} / m} v_{j}
$$

Then

$$
\begin{aligned}
\sum_{j=1}^{n} \int_{\mathbb{Z}_{m}^{n}}\left\|f\left(x+e_{j}\right)-f(x)\right\|_{X}^{p} d \mu(x) & =\left|e^{2 \pi i / m}-1\right|^{p} \cdot \sum_{j=1}^{n}\left\|v_{j}\right\|_{X}^{p} \\
& \leqslant\left(\frac{2 \pi}{m}\right)^{p} \cdot \sum_{j=1}^{n}\left\|v_{j}\right\|_{X}^{p},
\end{aligned}
$$

and

$$
\mathbb{E}_{\varepsilon} \int_{\mathbb{Z}_{m}^{n}}\left\|f\left(x+\frac{m}{2} \varepsilon\right)-f(x)\right\|_{X}^{p} d \mu(x)=2^{p} \int_{\mathbb{Z}_{m}^{n}}\left\|\sum_{j=1}^{n} e^{2 \pi i x_{j} / m} v_{j}\right\|_{X}^{p} d \mu(x) .
$$

We recall the contraction principle (see $[6]$ ), which states that for every $a_{1}, \ldots, a_{n} \in \mathbb{R}$,

$$
\mathbb{E}_{\varepsilon}\left\|\sum_{j=1}^{n} \varepsilon_{j} a_{j} v_{j}\right\|_{X}^{p} \leqslant\left(\max _{1 \leqslant j \leqslant n}\left|a_{j}\right|\right)^{p} \cdot \mathbb{E}_{\varepsilon}\left\|\sum_{j=1}^{n} \varepsilon_{j} v_{j}\right\|_{X}^{p} .
$$

Thus

$$
\begin{aligned}
\int_{\mathbb{Z}_{m}^{n}}\left\|\sum_{j=1}^{n} e^{2 \pi i x_{j} / m} v_{j}\right\|_{X}^{p} d \mu(x) & =\int_{\mathbb{Z}_{m}^{n}} \mathbb{E}_{\varepsilon}\left\|\sum_{j=1}^{n} e^{(2 \pi i / m)\left(x_{j}+m\left(1-\varepsilon_{j}\right) / 4\right)} v_{j}\right\|_{X}^{p} d \mu(x) \\
& =\int_{\mathbb{Z}_{m}^{n}} \mathbb{E}_{\varepsilon}\left\|\sum_{j=1}^{n} \varepsilon_{j} e^{2 \pi i x_{j} / m} v_{j}\right\|_{X}^{p} d \mu(x) \\
& \geqslant \frac{1}{2^{p}} \mathbb{E}_{\varepsilon}\left\|\sum_{j=1}^{n} \varepsilon_{j} v_{j}\right\|_{X}^{p}
\end{aligned}
$$

Combining (2.1), (2.2) and (2.3) yields the required result.

Let $X$ be a Banach space with type $p, m$ an integer divisible by 4 , and $k$ an odd integer. Fix $f: \mathbb{Z}_{m}^{n} \rightarrow X$ and $\varepsilon \in\{-1,1\}^{n}$. Define $\mathcal{A}^{(k)} f: \mathbb{Z}_{m}^{n} \rightarrow X$ by

$$
\mathcal{A}^{(k)} f(x)=\frac{1}{k^{n}} \sum_{z \in(-k, k)^{n} \cap(2 \mathbb{Z})^{n}} f(x+z) .
$$

Lemma 2.2. For $p \geqslant 1$ and every $f: \mathbb{Z}_{m}^{n} \rightarrow X$, we have

$$
\int_{\mathbb{Z}_{m}^{n}}\left\|\mathcal{A}^{(k)} f(x)-f(x)\right\|_{X}^{p} d \mu(x) \leqslant(k-1)^{p} n^{p-1} \sum_{j=1}^{n} \int_{\mathbb{Z}_{m}^{n}}\left\|f\left(x+e_{j}\right)-f(x)\right\|_{X}^{p} d \mu(x) .
$$

Proof. For every $t \in \mathbb{R}$ let $s(t)$ be the sign of $t$ (with the convention that $s(0)=0$ ). For every $z \in \mathbb{Z}_{m}^{n}$, we have

$$
\begin{aligned}
& \|f(x+z)-f(x)\|_{X}^{p} \\
& \leqslant\|z\|_{1}^{p-1} \cdot \sum_{j=1}^{n} \sum_{\ell=1}^{\left|z_{j}\right|}\left\|f\left(x+\sum_{t=1}^{j-1} z_{t} e_{t}+\ell \cdot s\left(z_{j}\right) \cdot e_{j}\right)-f\left(x+\sum_{t=1}^{j-1} z_{t} e_{t}+(\ell-1) \cdot s\left(z_{j}\right) \cdot e_{j}\right)\right\|_{X}^{p} .
\end{aligned}
$$


Observe that since $k$ is odd, $\left|(-k, k)^{n} \cap(2 \mathbb{Z})^{n}\right|=k^{n}$. Thus

$$
\begin{aligned}
& \int_{\mathbb{Z}_{m}^{n}}\left\|\mathcal{A}^{(k)} f(x)-f(x)\right\|_{X}^{p} d \mu(x) \\
& \leqslant \frac{1}{k^{n}} \sum_{z \in(-k, k)^{n} \cap(2 \mathbb{Z})^{n}} \int_{\mathbb{Z}_{m}^{n}}\|f(x+z)-f(x)\|_{X}^{p} d \mu(x) \\
& \leqslant \frac{1}{k^{n}} \sum_{z \in(-k, k)^{n} \cap(2 \mathbb{Z})^{n}} \int_{\mathbb{Z}_{m}^{n}}\|z\|_{1}^{p-1} \sum_{j=1}^{n} \sum_{\ell=1}^{\left|z_{j}\right|} \| f\left(x+\sum_{t=1}^{j-1} z_{t} e_{t}+\ell s\left(z_{j}\right) e_{j}\right) \\
& \leqslant \frac{1}{k^{n}} \sum_{z \in(-k, k)^{n} \cap(2 \mathbb{Z})^{n}} \sum_{j=1}^{n}\|z\|_{1}^{p-1}\left|z_{j}\right| \int_{\mathbb{Z}_{m}^{n}}\left\|f\left(x+\sum_{t=1}^{j-1} z_{t} e_{t}+(\ell-1) s\left(z_{j}\right) e_{j}\right)\right\|_{X}^{p} d \mu(x)-f(y) \|_{X}^{p} d \mu(x) \\
& \leqslant(k-1)^{p} n^{p-1} \sum_{j=1}^{n} \int_{\mathbb{Z}_{m}^{n}}\left\|f\left(x+e_{j}\right)-f(x)\right\|_{X}^{p} d \mu(x) .
\end{aligned}
$$

This completes the proof of Lemma 2.2.

Proof of Theorem 1.1. Fix an odd integer $k \in \mathbb{N}$, with $k<m / 2$. As in [8], given $j \in$ $\{1, \ldots, n\}$ we define $S(j, k) \subseteq \mathbb{Z}_{m}^{n}$ by

$$
S(j, k):=\left\{x \in[-k, k]^{n} \subseteq \mathbb{Z}_{m}^{n}: y_{j} \equiv 0(\bmod 2) \text { and } \forall \ell \neq j, y_{\ell} \equiv 1(\bmod 2)\right\}
$$

For $f: \mathbb{Z}_{m}^{n} \rightarrow X$, define

$$
\begin{aligned}
\mathcal{E}_{j}^{(k)} f(x) & =\left(f * \frac{\mathbf{1}_{S(j, k)}}{\mu(S(j, k))}\right)(x) \\
& =\frac{1}{\mu(S(j, k))} \int_{S(j, k)} f(x+y) d \mu(y) .
\end{aligned}
$$

In [8] (see equation (39) there) it is shown that for every $x \in \mathbb{Z}_{m}^{n}$ and $\varepsilon \in\{-1,1\}^{n}$,

$$
\begin{aligned}
\left(\frac{k}{k+1}\right)^{n-1}\left(\mathcal{A}^{(k)} f(x+\varepsilon)-\right. & \left.\mathcal{A}^{(k)} f(x-\varepsilon)\right) \\
& =\sum_{j=1}^{n} \varepsilon_{j}\left[\mathcal{E}_{j}^{(k)} f\left(x+e_{j}\right)-\mathcal{E}_{j}^{(k)} f\left(x-e_{j}\right)\right]+U(x, \varepsilon)+V(x, \varepsilon),
\end{aligned}
$$

where, by $\left[8\right.$, inequalities (41) and (42)], for every $\varepsilon \in\{-1,1\}^{n}$,

$$
\begin{aligned}
\max \left\{\int_{\mathbb{Z}_{m}^{n}}\|U(x, \varepsilon)\|_{X}^{p} d \mu(x), \int_{\mathbb{Z}_{m}^{n}}\|V(x, \varepsilon)\|_{X}^{p} d \mu(x)\right\} & \\
& \leqslant \frac{8^{p} n^{2 p-1}}{k^{p}} \sum_{j=1}^{n} \int_{\mathbb{Z}_{m}^{n}}\left\|f\left(x+e_{j}\right)-f(x)\right\|_{X}^{p} .
\end{aligned}
$$


Thus, for every $T>T_{p}(X)$, we have

$$
\begin{aligned}
&\left(\frac{k}{k+1}\right)^{p(n-1)} \mathbb{E}_{\varepsilon} \int_{\mathbb{Z}_{m}^{n}}\left\|\mathcal{A}^{(k)} f(x+\varepsilon)-\mathcal{A}^{(k)} f(x-\varepsilon)\right\|_{X}^{p} d \mu(x) \\
& \leqslant 3^{p-1} \mathbb{E}_{\varepsilon} \int_{\mathbb{Z}_{m}^{n}}\left\|\sum_{j=1}^{n} \varepsilon_{j}\left[\mathcal{E}_{j}^{(k)} f\left(x+e_{j}\right)-\mathcal{E}_{j}^{(k)} f\left(x-e_{j}\right)\right]\right\|_{X}^{p} d \mu(x) \\
&+\frac{24^{p} n^{2 p-1}}{k^{p}} \sum_{j=1}^{n} \int_{\mathbb{Z}_{m}^{n}}\left\|f\left(x+e_{j}\right)-f(x)\right\|_{X}^{p} d \mu(x) \\
& \leqslant 3^{p-1} T^{p} \sum_{j=1}^{n} \int_{\mathbb{Z}_{m}^{n}}\left\|\mathcal{E}_{j}^{(k)} f\left(x+e_{j}\right)-\mathcal{E}_{j}^{(k)} f\left(x-e_{j}\right)\right\|_{X}^{p} d \mu(x) \\
&+\frac{24^{p} n^{2 p-1}}{k^{p}} \sum_{j=1}^{n} \int_{\mathbb{Z}_{m}^{n}}\left\|f\left(x+e_{j}\right)-f(x)\right\|_{X}^{p} d \mu(x) \\
& \leqslant 3^{p-1} T^{p} \sum_{j=1}^{n} \int_{\mathbb{Z}_{m}^{n}}\left\|f\left(x+e_{j}\right)-f\left(x-e_{j}\right)\right\|_{X}^{p} d \mu(x) \\
&+\frac{24^{p} n^{2 p-1}}{k^{p}} \sum_{j=1}^{n} \int_{\mathbb{Z}_{m}^{n}}\left\|f\left(x+e_{j}\right)-f(x)\right\|_{X}^{p} d \mu(x) \\
& \leqslant\left(\frac{6^{p}}{3} T^{p}+\frac{24^{p} n^{2 p-1}}{k^{p}}\right) \sum_{j=1}^{n} \int_{\mathbb{Z}_{m}^{n}}\left\|f\left(x+e_{j}\right)-f(x)\right\|_{X}^{p} d \mu(x),
\end{aligned}
$$

where in $(2.5)$ we used the fact that $\mathcal{E}_{j}^{(k)}$ is an averaging operator, and hence has norm 1.

On the other hand

$$
\begin{aligned}
& \mathbb{E}_{\varepsilon} \int_{\mathbb{Z}_{m}^{n}}\left\|f\left(x+\frac{m}{2} \varepsilon\right)-f(x)\right\|_{X}^{p} d \mu(x) \\
& \leqslant 3^{p-1} \mathbb{E}_{\varepsilon} \int_{\mathbb{Z}_{m}^{n}}\left\|\mathcal{A}^{(k)} f\left(x+\frac{m}{2} \varepsilon\right)-\mathcal{A}^{(k)} f(x)\right\|_{X}^{p} d \mu(x) \\
& \quad+3^{p-1} \mathbb{E}_{\varepsilon} \int_{\mathbb{Z}_{m}^{n}}\left\|f\left(x+\frac{m}{2} \varepsilon\right)-\mathcal{A}^{(k)} f\left(x+\frac{m}{2} \varepsilon\right)\right\|_{X}^{p} d \mu(x) \\
& \quad+3^{p-1} \mathbb{E}_{\varepsilon} \int_{\mathbb{Z}_{m}^{n}}\left\|\mathcal{A}^{(k)} f(x)-f(x)\right\|_{X}^{p} d \mu(x) \\
& \leqslant 3^{p-1}\left[\left(\frac{m}{4}\right)^{p-1} \mathbb{E}_{\varepsilon} \int_{\mathbb{Z}_{m}^{n}} \sum_{t=1}^{m / 4}\left\|\mathcal{A}^{(k)} f(x+2 t \varepsilon)-\mathcal{A}^{(k)} f(x+(2 t-2) \varepsilon)\right\|_{X}^{p} d \mu(x)\right. \\
&\left.\quad+2 \mathbb{E}_{\varepsilon} \int_{\mathbb{Z}_{m}^{n}}\left\|\mathcal{A}^{(k)} f(x)-f(x)\right\|_{X}^{p} d \mu(x)\right] \\
& \leqslant 3^{p-1}\left[\left(\frac{m}{4}\right)^{p} \mathbb{E}_{\varepsilon} \int_{\mathbb{Z}_{m}^{n}} \sum_{t=1}^{m / 4}\left\|\mathcal{A}^{(k)} f(y+\varepsilon)-\mathcal{A}^{(k)} f(x-\varepsilon)\right\|_{X}^{p} d \mu(x)\right. \\
& \leqslant \quad\left[3^{p-1}\left(\frac{m}{4}\right)^{p}\left(1+\frac{1}{k}\right)^{n} n^{p-1} \sum_{j=1}^{n} \int_{\mathbb{Z}_{m}^{n}}^{p(n-1)}\left(\frac{6^{p}}{3} T^{p}+\frac{24^{p} n^{2 p-1}}{k^{p}}\right)+\frac{2(3 k n)^{p}}{3 n}\right] \\
& \cdot \sum_{j=1}^{n} \int_{\mathbb{Z}_{m}^{n}}\left\|f\left(x+e_{j}\right)-f(x)\right\|_{X}^{p} d \mu(x)
\end{aligned}
$$




$$
\leqslant 5^{p} m^{p} T^{p} \sum_{j=1}^{n} \int_{\mathbb{Z}_{m}^{n}}\left\|f\left(x+e_{j}\right)-f(x)\right\|_{X}^{p} d \mu(x),
$$

where in (2.7) we used Lemma 2.2, in (2.8) we used (2.6), and (2.9) is true provided that $4 n^{2-1 / p} \leqslant k \leqslant 3 m / 2 n^{1-1 / p}$, which is a valid choice of $k$ if $m \geqslant 3 n^{3-2 / p}$.

\section{References}

1. K. BALl, 'Markov chains, Riesz transforms and Lipschitz maps', Geom. Funct. Anal. 2 (1992) 137-172.

2. J. Bourgain, 'The metrical interpretation of superreflexivity in Banach spaces', Israel J. Math. 56 (1986) $222-230$.

3. J. Bourgain, V. Milman and H. Wolfson, 'On type of metric spaces', Trans. Amer. Math. Soc. 294 (1986) 295-317.

4. D. L. Burkholder, 'Martingales and singular integrals in Banach spaces', Handbook of the geometry of Banach spaces, vol. 1 (ed. W. B. Johnson and J. Lindenstrauss; North-Holland, Amsterdam, 2001) $233-269$.

5. P. Enflo, 'On infinite-dimensional topological groups', Séminaire sur la Géométrie des Espaces de Banach, 1977-1978 (École Polytech., Palaiseau, 1978) Exp. No. 10-11, 11.

6. M. Ledoux and M. Talagrand, Probability in Banach spaces. Isoperimetry and processes, Ergeb. Math. Grenzgeb. (3) 23 (Springer, Berlin, 1991).

7. B. Maurey, 'Type, cotype and $K$-convexity', Handbook of the geometry of Banach spaces, vol. 2 (ed. W. B. Johnson and J. Lindenstrauss; North-Holland, Amsterdam, 2003) 1299-1332.

8. M. Mendel and A. NAor, 'Metric cotype', Ann. of Math., to appear.

9. A. Naor, Y. Peres, O. Schramm and S. Sheffield, 'Markov chains in smooth Banach spaces and Gromov hyperbolic metric spaces', Duke Math. J. 134 (2006) 165-197.

10. A. NAor and G. Schechtman, 'Remarks on non linear type and Pisier's inequality', J. Reine Angew. Math. 552 (2002) 213-236.

11. G. Pisien, 'Probabilistic methods in the geometry of Banach spaces', Probability and analysis, Varenna, 1985, Lecture Notes in Math. 1206 (Springer, Berlin, 1986) 167-241.

12. M. RiBe, 'On uniformly homeomorphic normed spaces', Ark. Mat. 14 (1976) 237-244.

Manor Mendel

California Institute of Technology

Pasadena, $C A$

USA

Current Address:

The Open University of Israel

108 Ravutzki St.

Ra'anana 43107

Israel

mendelma@gmail.com

\section{Assaf Naor \\ Microsoft Research \\ One Microsoft Way \\ Redmond WA 98052 \\ USA}

anaor@microsoft.com

Current Address:

New York University

251 Mercer Street

New York, NY 10012

USA

naor@cims.nyu.edu 